\title{
Convergence of Hybrid Steepest-Descent Methods for Generalized Variational Inequalities
}

\author{
L. C. Zeng ${ }^{1}$, N. C. Wong ${ }^{2}$ and J. C. Yao ${ }^{3}$
}

\footnotetext{
${ }^{1}$ Department of Mathematics, Shanghai Normal University, Shanghai 200234, China. This research was partially supported by the Teaching and Research Award Fund for Outstanding Young Teachers in Higher Education Institutions of MOE, China and the Dawn Program Foundation in Shanghai.

${ }^{2}$ Department of Applied Mathematics, National Sun Yat-sen University, Kaohsiung, Taiwan 804. This research was partially supported by grant from the National Science Council of Taiwan.

${ }^{3}$ Department of Applied Mathematics, National Sun Yat-sen University, 804 Kaohsiung, Taiwan. This research was partially supported by a grant from the National Science Council of Taiwan.
} 
Abstract. In this paper we consider the general variational inequality $\operatorname{GVI}(F, g, C)$ where $F$ and $g$ are mappings from a Hilbert space into itself and $C$ is the fixed points set of a nonexpansive mapping. We propose two iterative algorithms to find approximate solutions of the $\operatorname{GVI}(F, g, C)$. Strong convergence results are established and applications to constrained generalized pseudoinverse are included.

Key Words. Iterative algorithms, hybrid steepest-descent methods, nonexpansive mappings, Hilbert space, constrained generalized pseudoinverse.

2000 Mathematics Subject Classification. 49J30, 47H09, 47H10. 


\section{Introduction}

Let $H$ be a real Hilbert space with inner product $\langle\cdot, \cdot\rangle$ and norm $\|\cdot\|$, respectively. Let $C$ be a nonempty closed convex subset of $H$ and let $F: H \rightarrow H$ be a nonlinear operator and let $g: H \rightarrow H$ be a continuous mapping, respectively. We consider in this paper the following problem of finding a point $u^{*} \in H$ such that $g\left(u^{*}\right) \in C$ and

$$
\operatorname{GVI}(F, g, C): \quad\left\langle F\left(u^{*}\right), v-g\left(u^{*}\right)\right\rangle \geq 0, \quad \forall v \in C
$$

The above problem was studied by Pang and Yao (Ref. 12) by employing the concept of generalized normal map.

If $g$ is the identity mapping of $H$, then the $\operatorname{GVI}(F, g, C)$ reduces to finding a point $u^{*} \in C$ such that

$$
\mathrm{VI}(F, C): \quad\left\langle F\left(u^{*}\right), v-u^{*}\right\rangle \geq 0, \quad \forall v \in C
$$

which is called a variational inequality. Variational inequalities were initially studied by Stampacchia (cf. Ref. 8) and subsequently have been being widely studied because they cover various problems such as partial differential equations, optimal control, optimization, mathematical programming, mechanics and finance. The reader is referred to Refs. 6-8, 11, 18 and the references therein. 
It is known that when $F$ is strongly monotone on $C$, the $\operatorname{VI}(F, C)$ has a unique solution, e.g. see Ref. 18. Moreover, a great deal of effort has been devoted to the study of constructively iterative algorithms to find approximate solutions of $\mathrm{VI}(F, C)$, e.g., see Refs. 6 and 9.

On the other hand it is easy to see that the $\operatorname{GVI}(F, g, C)$ is equivalent to the fixed-point problem

$$
u^{*}=u^{*}-g\left(u^{*}\right)+P_{C}\left(g\left(u^{*}\right)-\mu F\left(u^{*}\right)\right)
$$

where $\mu>0$ is an arbitrarily fixed constant and $P_{C}$ is the (nearest point) projection from $H$ onto $C$, i.e., $P_{C} x=\arg \min _{y \in C}\|x-y\|$ for each $x \in H$. If $F$ and $g$ are strongly monotone and Lipschitzian on $C$ and $\mu>0$ is small enough, then the mapping determined by the right-hand side of this equation is a contraction. Hence the Banach contraction principle guarantees that the Picard iterates converge in norm to the unique solution of the $\operatorname{GVI}(F, g, C)$. Such a method is called the projection method. It has been widely extended to develop various algorithms for finding solutions of various classes of variational inequalities and complementarity problems; see Zeng (Refs. 19-21). It is remarkable that the fixed-point equation involves the projection $P_{C}$ which may not be easy to compute due to the complexity of the convex set $C$.

Recently Yamada (Ref. 17, see also Ref. 3) introduced a hybrid steepest-descent method for solving the $\operatorname{VI}(F, C)$ to reduce the complexity probably caused by the projection $P_{C}$. His idea is stated now. Let $C$ be the fixed point set of a nonexpansive mapping $T: H \rightarrow H$; that is, $C=\operatorname{Fix}(T)=\{x \in H: T x=x\}$. Recall that $T$ is nonexpansive if $\|T x-T y\| \leq\|x-y\|$ 
for all $x, y \in H$. Let $F$ be $\eta$-strongly monotone and $\kappa$-Lipschitzian on $C$. Take a fixed number $\mu \in\left(0,2 \eta / \kappa^{2}\right)$ and a sequence $\left\{\lambda_{n}\right\}$ of real numbers in $(0,1)$ satisfying the conditions below:

(L1) $\lim _{n \rightarrow \infty} \lambda_{n}=0$

(L2) $\sum_{n=1}^{\infty} \lambda_{n}=\infty$

(L3) $\lim _{n \rightarrow \infty}\left(\lambda_{n}-\lambda_{n+1}\right) / \lambda_{n+1}^{2}=0$.

Starting with an arbitrary initial guess $u_{0} \in H$, one can generate a sequence $\left\{u_{n}\right\}$ by the following algorithm:

$$
u_{n+1}:=T u_{n}-\lambda_{n+1} \mu F\left(T u_{n}\right), \quad n \geq 0 .
$$

Then Yamada (Ref. 17) proved that $\left\{u_{n}\right\}$ converges strongly to the unique solution of the $\mathrm{VI}(F, C)$. An example of the sequence $\left\{\lambda_{n}\right\}$ which satisfies conditions (L1)-(L3) is given by $\lambda_{n}=1 / n^{\sigma}$ where $0<\sigma<1$. Note that condition (L3) was first used by Lions (Ref. 10). Furthermore if $C$ is expressed as the intersection of the fixed-point sets of $N$ nonexpansive mappings $T_{i}: H \rightarrow H$ with $N \geq 1$ an integer, Yamada (Ref. 17) proposed another algorithm,

$$
u_{n+1}:=T_{[n+1]} u_{n}-\lambda_{n+1} \mu F\left(T_{[n+1]} u_{n}\right), \quad n \geq 0
$$

where $T_{[k]}:=T_{k \bmod N}$ for integer $k \geq 1$ with the mod function taking values in the set $\{1,2, \ldots, N\}$; that is, if $k=j N+q$ for some integers $j \geq 0$ and $0 \leq q<N$, then $T_{[k]}=T_{N}$ if $q=0$ and $T_{[k]}=T_{q}$ if $1 \leq q<N$, where $\mu \in\left(0,2 \eta / \kappa^{2}\right)$ and where the sequence $\left\{\lambda_{n}\right\}$ of parameters satisfies conditions (L1), (L2), and (L4) stated below:

(L4) $\sum_{n=1}^{\infty}\left|\lambda_{n}-\lambda_{n+N}\right|$ is convergent. 
Under these conditions Yamada (Ref. 17) proved the strong convergence of $\left\{u_{n}\right\}$ to the unique solution of the $\mathrm{VI}(F, C)$. Note that condition (L4) was first used by Bauschke (Ref. 1). In the special case of $N=1,(\mathrm{~L} 4)$ was introduced by Wittmann (Ref. 13).

In 2003, Xu and Kim (Ref. 16) further considered and studied the hybrid steepest-descent algorithms (1) and (2). Their major contribution is that the strong convergence of algorithms (1) and (2) holds with condition (L3) replaced by the condition

(L3)' $\lim _{n \rightarrow \infty} \lambda_{n} / \lambda_{n+1}=1$ or equivalently $\lim _{n \rightarrow \infty}\left(\lambda_{n}-\lambda_{n+1}\right) / \lambda_{n+1}=0$, and with condition (L4) replaced by the condition

(L4)' $\lim _{n \rightarrow \infty} \lambda_{n} / \lambda_{n+N}=1$ or equivalently $\lim _{n \rightarrow \infty}\left(\lambda_{n}-\lambda_{n+N}\right) / \lambda_{n+N}=0$.

It is clear that condition (L3)' is strictly weaker than condition (L3), coupled with conditions (L1) and (L2). Moreover (L3)' includes the important and natural choice $\{1 / n\}$ for $\left\{\lambda_{n}\right\}$ while (L3) does not. It is easy to see that if $\lim _{n \rightarrow \infty} \lambda_{n} / \lambda_{n+N}$ exists then condition (L4) implies condition (L4)'. However in general, conditions (L4) and (L4)' are not comparable: neither of them implies the other (see Ref. 15 for details). In addition, under conditions (L1), (L2), (L3)' and (L4)', they gave the applications of algorithms (1) and (2) to the constrained generalized pseudoinverses.

Remark 1.1. Although algorithms (1) and (2) have successfully been applied to finding the unique solution of the $\operatorname{VI}(F, C)$, it is clear that they can not be directly applied to computing solutions of the $\operatorname{GVI}(F, g, C)$ due to the appearance of $g$. Therefore, an important 
problem is how to apply hybrid steepest-descent methods to solving the $\operatorname{GVI}(F, g, C)$.

Remark 1.2. In the case when $H=R^{n}$ the $n$-dimensional Euclidean space, we can give a nontrival example; that is, the unique solution $u^{*}$ of the $\operatorname{VI}(F, C)$ is also a fixed point of a mapping $g: H \rightarrow H$ which is Lipschitzian and $\delta$-strongly monotone on $R^{n}$ with $\delta>1$. Indeed at first let $u^{*}=\left(a_{1}^{*}, a_{2}^{*}, \ldots, a_{n}^{*}\right) \in R^{n}$ be the unique solution of the $\operatorname{VI}(F, C)$.

Case 1: $a_{i}^{*} \neq 0,1 \leq i \leq n$. Put $r=\max \left\{\left|\frac{\pi}{2 a_{i}^{*}}\right|: 1 \leq i \leq n\right\}$ and define $g: H \rightarrow H$ by

$$
g(x)=\left(\frac{3}{2}+r\right) x-m(x)+x_{0} \quad \forall x=\left(a_{1}, a_{2}, \ldots, a_{n}\right) \in R^{n},
$$

where $x_{0}=\left(1-\left(\frac{1}{2}+r\right) a_{1}^{*}, 1-\left(\frac{1}{2}+r\right) a_{2}^{*}, \ldots, 1-\left(\frac{1}{2}+r\right) a_{n}^{*}\right)$ and

$$
m(x)=\left(\sin \left(\frac{\pi a_{1}}{2 a_{1}^{*}}\right), \sin \left(\frac{\pi a_{2}}{2 a_{2}^{*}}\right), \ldots, \sin \left(\frac{\pi a_{n}}{2 a_{n}^{*}}\right)\right) .
$$

It is easy to see that $u^{*}$ is a fixed point of $g$. Now observe that for all $x, y \in R^{n}, x=$

$$
\begin{aligned}
\left(a_{1}, a_{2}, \ldots, a_{n}\right), y=\left(b_{1}, b_{2}, \ldots, b_{n}\right), & \\
\|m(x)-m(y)\| & =\sqrt{\sum_{i=1}^{n}\left(\sin \left(\frac{\pi a_{i}}{2 a_{i}^{*}}\right)-\sin \left(\frac{\pi b_{i}}{2 a_{i}^{*}}\right)\right)^{2}} \\
& \leq \sqrt{\sum_{i=1}^{n}\left(\frac{\pi}{2 a_{i}^{*}}\right)^{2}\left(a_{i}-b_{i}\right)^{2}} \leq r\|x-y\|
\end{aligned}
$$

and hence

$$
\langle g(x)-g(y), x-y\rangle=\left(\frac{3}{2}+r\right)\|x-y\|^{2}-\langle m(x)-m(y), x-y\rangle \geq \frac{3}{2}\|x-y\|^{2} .
$$

This shows that $g$ is $\delta$-strongly monotone with $\delta=\frac{3}{2}$. Obviously $g$ is Lipschitzian on $R^{n}$.

Case 2: $a_{i_{0}}^{*}=0$ and $a_{j_{0}}^{*} \neq 0$ for some $i_{0}, j_{0}$. Without loss of generality, we may assume that $a_{i}^{*}=0,1 \leq i \leq k$ where $k<n$. Then put $r=\max \left\{\left|\frac{\pi}{2 a_{i}^{*}}\right|: k+1 \leq i \leq n\right\}$ and define 
$g: H \rightarrow H$ by

$$
g(x)=\left(\frac{3}{2}+r\right) x-m(x)+x_{0}, \quad \forall x=\left(a_{1}, a_{2}, \ldots, a_{n}\right) \in R^{n}
$$

where $x_{0}=\left(0, \ldots, 0,1-\left(\frac{1}{2}+r\right) a_{k+1}^{*}, \ldots, 1-\left(\frac{1}{2}+r\right) a_{n}^{*}\right)$ and

$$
m(x)=\left(0, \ldots, 0, \sin \left(\frac{\pi a_{k+1}}{2 a_{k+1}^{*}}\right), \ldots, \sin \left(\frac{\pi a_{n}}{2 a_{n}^{*}}\right) .\right.
$$

It is easy to see that $u^{*}$ is a fixed point of the mapping $g$ which is Lipschitzian and $\delta$-strongly monotone on $R^{n}$ with $\delta=\frac{3}{2}$.

Case 3: $a_{i}^{*}=0,1 \leq i \leq n$. Define $g: H \rightarrow H$ by

$$
g(x)=2 x-m(x) \quad \forall x=\left(a_{1}, a_{2}, \ldots, a_{n}\right) \in R^{n}
$$

where $m(x)=\left(\frac{1}{2} \sin a_{1}, \frac{1}{2} \sin a_{2}, \ldots, \frac{1}{2} \sin a_{n}\right)$. It is also easy to see that $u^{*}$ is a fixed point of the mapping $g$ which is Lipschitzian and $\delta$-strongly monotone on $R^{n}$ with $\delta=\frac{3}{2}$.

In this paper motivated and inspired by algorithms (1) and (2), we introduce the following hybrid steepest-descent algorithms (I) and (II) for $\operatorname{GVI}(F, g, C)$.

Algorithm (I). Let $\left\{\lambda_{n}\right\} \subset(0,1),\left\{\theta_{n}\right\} \subset(0,1]$ and $\mu \in\left(0,2 \eta / \kappa^{2}\right)$. Starting with an arbitrary initial guess $u_{0} \in H$, one can generate a sequence $\left\{u_{n}\right\}$ by the following iterative scheme

$$
u_{n+1}:=\left(1+\theta_{n+1}\right) T u_{n}-\theta_{n+1} g\left(T u_{n}\right)-\lambda_{n+1} \mu F\left(T u_{n}\right) \quad n \geq 0 .
$$

Algorithm (II). Let $\left\{\lambda_{n}\right\} \subset(0,1),\left\{\theta_{n}\right\} \subset(0,1]$ and $\mu \in\left(0,2 \eta / \kappa^{2}\right)$. Starting with an 
arbitrary initial guess $u_{0} \in H$, one can generate a sequence $\left\{u_{n}\right\}$ by the following iterative scheme

$$
u_{n+1}:=\left(1+\theta_{n+1}\right) T_{[n+1]} u_{n}-\theta_{n+1} g\left(T_{[n+1]} u_{n}\right)-\lambda_{n+1} \mu F\left(T_{[n+1]} u_{n}\right) \quad n \geq 0 .
$$

Remark 1.3. If $g=I$ the identity mapping of $H$, then Algorithms (I) and (II) reduce to algorithms (1) and (2), respectively. Therefore algorithms (1) and (2) are special cases of our Algorithms (I) and (II), respectively. For simplicity we discuss the convergence of Algorithms (I) and (II) only in the case where $\delta>1$.

Throughout this paper, let $g$ be $\sigma$-Lipschitzian and $\delta$-strongly monotone on $C$ such that $\delta>1$ and let the unique solution $u^{*}$ of the $\operatorname{VI}(F, C)$ be a fixed point of $g$. Assume that $\left\{\theta_{n}\right\}$ satisfies the restrictions (R1), (R2), (R3) or the ones (R1), (R2), (R4) where (R1), (R2), (R3) and (R4) are defined as follows:

$$
\begin{aligned}
& (\mathrm{R} 1)\left\{\theta_{n}\right\} \subset\left(0,2(\delta-1) /\left(\sigma^{2}-1\right)\right] \\
& (\mathrm{R} 2) \lim _{n \rightarrow \infty} \theta_{n}=0, \lim _{n \rightarrow \infty} \lambda_{n} / \theta_{n}=0 ; \\
& \text { (R3) } \sum_{n=1}^{\infty}\left|\theta_{n+1}-\theta_{n}\right|<\infty ; \\
& \text { (R4) } \sum_{n=1}^{\infty}\left|\theta_{n+N}-\theta_{n}\right|<\infty .
\end{aligned}
$$

Firstly under conditions (L1), (L2), (L3)' and restrictions (R1), (R2), (R3), we prove that the sequence $\left\{u_{n}\right\}$ generated by Algorithm (I) converges in norm to the unique solution of the $\mathrm{VI}(F, C)$ (i.e., a solution of the $\operatorname{GVI}(F, g, C))$. 
Secondly under conditions (L1), (L2), (L4)' and restrictions (R1), (R2), (R4), we prove that the sequence $\left\{u_{n}\right\}$ generated by Algorithm (II) converges in norm to the unique solution of the $\operatorname{VI}(F, C)$ (i.e., a solution of the $\operatorname{GVI}(F, g, C)$ ). Furthermore we give applications of these two results to constrained generalized pseudoinverse.

\section{Preliminaries}

The following lemma will be used in the proofs of the main results of this paper in Section 3.

Lemma 2.1. (See Lemma 2.5 in Ref. 14.) Let $\left\{s_{n}\right\}$ be a sequence of nonnegative real numbers satisfying

$$
s_{n+1} \leq\left(1-\alpha_{n}\right) s_{n}+\alpha_{n} \beta_{n}+\gamma_{n}, \quad \forall n \geq 0,
$$

where $\left\{\alpha_{n}\right\},\left\{\beta_{n}\right\}$, and $\left\{\gamma_{n}\right\}$ satisfy the conditions:

(i) $\left\{\alpha_{n}\right\} \subset[0,1], \sum_{n=0}^{\infty} \alpha_{n}=+\infty$;

(ii) $\lim _{n \rightarrow \infty} \beta_{n} \leq 0$;

(iii) $\gamma_{n} \geq 0(\forall n \geq 0), \quad \sum_{n=0}^{\infty} \gamma_{n}<+\infty$.

Then $\lim _{n \rightarrow \infty} s_{n}=0$.

Lemma 2.2. Demiclosedness Principle. (See Ref. 5.) Assume that $T$ is a nonexpansive self-mapping of a closed convex subset $C$ of a Hilbert space $H$. If $T$ has a fixed point, then 
$I-T$ is demiclosed; that is, whenever $\left\{x_{n}\right\}$ is a sequence in $C$ weakly converging to some $x \in C$ and the sequence $\left\{(I-T) x_{n}\right\}$ strongly converges to some $y$, it follows that $(I-T) x=y$.

Here $I$ is the identity operator of $H$.

The following lemma is an immediate consequence of an inner product.

Lemma 2.3. In a real Hilbert space $H$, there holds the inequality

$$
\|x+y\|^{2} \leq\|x\|^{2}+2\langle y, x+y\rangle \quad \forall x, y \in H
$$

\section{Convergence of Hybrid Steepest-Descent Algorithms}

Let $H$ be a real Hilbert space and let $C$ be a nonempty closed convex subset of $H$. Let $F: H \rightarrow H$ be an operator such that for some constants $\kappa, \eta>0, F$ is $\kappa$-Lipschitzian and $\eta$-strongly monotone on $C$; that is, $F$ satisfies the conditions

$$
\begin{gathered}
\|F x-F y\| \leq \kappa\|x-y\| \quad \forall x, y \in C, \\
\langle F x-F y, x-y\rangle \geq \eta\|x-y\|^{2} \quad \forall x, y \in C .
\end{gathered}
$$

Since $F$ is strongly monmotone, $\operatorname{VI}(F, C)$ has a unique solution $u^{*} \in C$.

Let $g: H \rightarrow H$ be a mapping such that $g$ is $\sigma$-Lipschitzian and $\delta$-strongly monotone on $C$ for some constants $\sigma>0$ and $\delta>1$. Assume also that the unique solution $u^{*}$ of the $\operatorname{VI}(F, C)$ is a fixed point of $g$. 
Denote by $P_{C}$ the projection of $H$ onto $C$. Namely for each $x \in H, P_{C} x$ is the unique element in $C$ satisfying

$$
\left\|x-P_{C} x\right\|=\min \{\|x-y\|: y \in C\} .
$$

It is known that the projection $P_{C}$ is characterized by the inequality

$$
\left\langle x-P_{C} x, y-P_{C} x\right\rangle \leq 0 \quad \forall y \in C .
$$

Thus it follows that the $\operatorname{VI}(F, C)$ is equivalent to the fixed-point problem

$$
u^{*}=P_{C}(I-\mu F) u^{*}
$$

where $\mu>0$ is an arbitrary constant.

In this section assume that $T: H \rightarrow H$ is a nonexpansive mapping with $\operatorname{Fix}(T)=C$. Note that obviously $\operatorname{Fix}\left(P_{C}\right)=C$. Let $0<\mu<2 \eta / \kappa^{2}$. For any given numbers $\lambda \in(0,1]$ and $\theta \in\left(0,2(\delta-1) /\left(\sigma^{2}-1\right)\right]$ associated with the nonexpansive mapping $T$ we define the mapping

$$
T^{(\theta, \lambda)} x:=(1+\theta) T x-\theta g(T x)-\lambda \mu F(T x) \quad \forall x \in H .
$$

Algorithm (I). Let $\left\{\lambda_{n}\right\} \subset(0,1)$, and $\left\{\theta_{n}\right\} \subset(0,1]$ and $\mu \in\left(0,2 \eta / \kappa^{2}\right)$. Starting with an arbitrary initial guess $u_{0} \in H$, one can generate a sequence $\left\{u_{n}\right\}$ by the following iterative scheme

$$
u_{n+1}:=T^{\left(\theta_{n+1} \lambda_{n+1}\right)} u_{n}=\left(1+\theta_{n+1}\right) T u_{n}-\theta_{n+1} g\left(T u_{n}\right)-\lambda_{n+1} \mu F\left(T u_{n}\right), \quad n \geq 0 .
$$


Theorem 3.1. Assume that $0<\mu<2 \eta / \kappa^{2}$. Assume that the control conditions (L1), (L2) and (L3)' hold for $\left\{\lambda_{n}\right\}$ and also that the restrictions (R1), (R2) and (R3) hold for $\left\{\theta_{n}\right\}$. Assume that $u^{*} \in \operatorname{Fix}(g)$. Then the sequence $\left\{u_{n}\right\}$ generated by algorithm (6) converges strongly to $u^{*}$ which is a solution of the $\operatorname{GVI}(F, g, C)$.

Proof. First, since $u^{*}$ is a solution of $\operatorname{VI}(F, C)$ and also a fixed point of $g, u^{*}$ is a solution of the $\mathrm{GVI}(F, g, C)$. We divide the proof into several steps.

Step 1. $\left\{u_{n}\right\}$ is bounded. Indeed, we have (note that $T^{\left(\theta_{n+1}, \lambda_{n+1}\right)} u^{*}=u^{*}-\lambda_{n+1} \mu F u^{*}$ since $\left.u^{*} \in \operatorname{Fix}(T) \cap \operatorname{Fix}(g)\right)$

$$
\begin{aligned}
\left\|u_{n+1}-u^{*}\right\| & =\left\|T^{\left(\theta_{n+1}, \lambda_{n+1}\right)} u_{n}-u^{*}\right\| \\
& \leq\left\|T^{\left(\theta_{n+1}, \lambda_{n+1}\right)} u_{n}-T^{\left(\theta_{n+1}, \lambda_{n+1}\right)} u^{*}\right\|+\left\|T^{\left(\theta_{n+1}, \lambda_{n+1}\right)} u^{*}-u^{*}\right\| \\
& \leq\left\|T^{\left(\theta_{n+1}, \lambda_{n+1}\right)} u_{n}-T^{\left(\theta_{n+1}, \lambda_{n+1}\right)} u^{*}\right\|+\lambda_{n+1} \mu\left\|F\left(u^{*}\right)\right\| .
\end{aligned}
$$

Observe that

$$
\begin{aligned}
& \left\|T u_{n}-T u^{*}-\theta_{n+1}\left(g\left(T u_{n}\right)-g\left(T u^{*}\right)\right)\right\|^{2} \\
& =\left\|T u_{n}-T u^{*}\right\|^{2}-2 \theta_{n+1}\left\langle g\left(T u_{n}\right)-g\left(T u^{*}\right), T u_{n}-T u^{*}\right\rangle \\
& \quad+\theta_{n+1}^{2}\left\|g\left(T u_{n}\right)-g\left(T u^{*}\right)\right\|^{2} \\
& \leq\left(1-2 \theta_{n+1} \delta+\theta_{n+1}^{2} \sigma^{2}\right)\left\|T u_{n}-T u^{*}\right\|^{2} \\
& \leq\left(1-2 \theta_{n+1} \delta+\theta_{n+1}^{2} \sigma^{2}\right)\left\|u_{n}-u^{*}\right\|^{2}
\end{aligned}
$$

and

$$
\begin{aligned}
& \left\|\theta_{n+1}\left(T u_{n}-T u^{*}\right)-\lambda_{n+1} \mu\left(F\left(T u_{n}\right)-F\left(T u^{*}\right)\right)\right\|^{2} \\
& =\theta_{n+1}^{2}\left\|T u_{n}-T u^{*}\right\|^{2}-2 \theta_{n+1} \lambda_{n+1} \mu\left\langle F\left(T u_{n}\right)-F\left(T u^{*}\right), T u_{n}-T u^{*}\right\rangle \\
& \quad+\left(\lambda_{n+1} \mu\right)^{2}\left\|F\left(T u_{n}\right)-F\left(T u^{*}\right)\right\|^{2} \\
& \leq\left(\theta_{n+1}^{2}-2 \theta_{n+1} \lambda_{n+1} \mu \eta+\left(\lambda_{n+1} \mu\right)^{2} \kappa^{2}\right)\left\|T u_{n}-T u^{*}\right\|^{2} \\
& \leq\left(\theta_{n+1}^{2}-2 \theta_{n+1} \lambda_{n+1} \mu \eta+\left(\lambda_{n+1} \mu\right)^{2} \kappa^{2}\right)\left\|u_{n}-u^{*}\right\|^{2} \\
& =\theta_{n+1}^{2}\left(1-\frac{2 \lambda_{n+1} \mu \eta}{\theta_{n+1}}+\frac{\left(\lambda_{n+1} \mu\right)^{2} \kappa^{2}}{\theta_{n+1}^{2}}\right)\left\|u_{n}-u^{*}\right\|^{2} \\
& =\theta_{n+1}^{2}\left[\left(1-\frac{\lambda_{n+1} \mu \kappa}{\theta_{n+1}}\right)^{2}+\frac{2 \lambda_{n+1} \mu(\kappa-\eta)}{\theta_{n+1}}\right]\left\|u_{n}-u^{*}\right\|^{2} .
\end{aligned}
$$

We have 


$$
\begin{aligned}
& \left\|T^{\left(\theta_{n+1}, \lambda_{n+1}\right)} u_{n}-T^{\left(\theta_{n+1}, \lambda_{n+1}\right)} u^{*}\right\| \\
& =\|\left(T u_{n}-T u^{*}-\theta_{n+1}\left(g\left(T u_{n}\right)-g\left(T u^{*}\right)\right)\right) \\
& +\left[\theta_{n+1}\left(T u_{n}-T u^{*}\right)-\lambda_{n+1} \mu\left(F\left(T u_{n}\right)-F\left(T u^{*}\right)\right)\right] \| \\
& \leq\left\|T u_{n}-T u^{*}-\theta_{n+1}\left(g\left(T u_{n}\right)-g\left(T u^{*}\right)\right)\right\| \\
& +\left\|\theta_{n+1}\left(T u_{n}-T u^{*}\right)-\lambda_{n+1} \mu\left(F\left(T u_{n}\right)-F\left(T u^{*}\right)\right)\right\| \\
& \leq \sqrt{1-2 \theta_{n+1} \delta+\theta_{n+1}^{2} \sigma^{2}}\left\|u_{n}-u^{*}\right\| \\
& +\theta_{n+1} \sqrt{\left(1-\frac{\lambda_{n+1} \mu \kappa}{\theta_{n+1}}\right)^{2}+\frac{2 \lambda_{n+1} \mu(\kappa-\eta)}{\theta_{n+1}}}\left\|u_{n}-u^{*}\right\| \\
& \leq \sqrt{1-2 \theta_{n+1} \delta+\theta_{n+1}^{2} \sigma^{2}}\left\|u_{n}-u^{*}\right\| \\
& +\theta_{n+1}\left|1-\frac{\lambda_{n+1} \mu \kappa}{\theta_{n+1}}\right| \sqrt{1+\left(\frac{2 \lambda_{n+1} \mu(\kappa-\eta)}{\theta_{n+1}}\right) /\left(1-\frac{\lambda_{n+1} \mu \kappa}{\theta_{n+1}}\right)^{2}}\left\|u_{n}-u^{*}\right\| .
\end{aligned}
$$

Now we can see that (R2) yields

$$
\lim _{n \rightarrow \infty}\left(\frac{\lambda_{n+1} \mu \kappa}{\theta_{n+1}}-\frac{\eta}{\kappa}\right) /\left(1-\frac{\lambda_{n+1} \mu \kappa}{\theta_{n+1}}\right)=-\frac{\eta}{\kappa} .
$$

Hence noticing (L1) and (R2), we infer that there exists an integer $N_{0} \geq 0$ such that for all $n \geq N_{0}$,

$$
\frac{1}{2} \lambda_{n+1} \mu \eta<1, \quad 1-\frac{\lambda_{n+1} \mu \kappa}{\theta_{n+1}}>0 \quad \text { and } \quad\left(\frac{\lambda_{n+1} \mu \kappa}{\theta_{n+1}}-\frac{\eta}{\kappa}\right) /\left(1-\frac{\lambda_{n+1} \mu \kappa}{\theta_{n+1}}\right)<-\frac{\eta}{2 \kappa}
$$

Thus we deduce that for all $n \geq N_{0}$,

$$
\begin{aligned}
& \theta_{n+1}\left|1-\frac{\lambda_{n+1} \mu \kappa}{\theta_{n+1}}\right| \sqrt{1+\left(\frac{2 \lambda_{n+1} \mu(\kappa-\eta)}{\theta_{n+1}}\right) /\left(1-\frac{\lambda_{n+1} \mu \kappa}{\theta_{n+1}}\right)^{2}} \\
& \leq \theta_{n+1}\left(1-\frac{\lambda_{n+1} \mu \kappa}{\theta_{n+1}}\right)\left(1+\left(\frac{\lambda_{n+1} \mu(\kappa-\eta)}{\theta_{n+1}}\right) /\left(1-\frac{\lambda_{n+1} \mu \kappa}{\theta_{n+1}}\right)^{2}\right) \\
& =\theta_{n+1}-\lambda_{n+1} \mu \kappa+\frac{\lambda_{n+1} \mu(\kappa-\eta)}{1-\frac{\lambda_{n+1} \mu \kappa}{\theta_{n+1}}} \\
& =\theta_{n+1}+\frac{-\lambda_{n+1} \mu \kappa+\frac{\left(\lambda_{n+1} \mu \kappa\right)^{2}}{\theta_{n+1}}+\lambda_{n+1} \mu \kappa-\lambda_{n+1} \mu \eta}{1-\frac{\lambda_{n+1} \mu \kappa}{\theta_{n+1}}} \\
& =\theta_{n+1}+\lambda_{n+1} \mu \kappa\left[\left(\frac{\lambda_{n+1} \mu \kappa}{\theta_{n+1}}-\frac{\eta}{\kappa}\right) /\left(1-\frac{\lambda_{n+1} \mu \kappa}{\theta_{n+1}}\right)\right] \\
& \leq \theta_{n+1}-\frac{1}{2} \lambda_{n+1} \mu \eta .
\end{aligned}
$$

Observe that $\sigma \geq \delta$ implies $1-2 \delta+\sigma^{2} \geq 0$ and hence $2(\delta-1) /\left(\sigma^{2}-1\right) \leq 1$. From $(\mathrm{R} 1)$, we obtain

$$
0<\theta_{n+1} \leq \frac{2(\delta-1)}{\sigma^{2}-1} \leq 1
$$


Therefpre we conclude that

$$
\begin{aligned}
0<\theta_{n+1} \leq \frac{2(\delta-1)}{\sigma^{2}-1} & \Leftrightarrow \theta_{n+1}^{2}\left(\sigma^{2}-1\right) \leq 2 \theta_{n+1}(\delta-1) \\
& \Leftrightarrow \theta_{n+1}^{2}\left(\sigma^{2}-1\right)+1 \leq 2 \theta_{n+1}(\delta-1)+1 \\
& \Leftrightarrow\left(1-\theta_{n+1}\right)^{2} \geq 1-2 \theta_{n+1} \delta+\theta_{n+1}^{2} \sigma^{2} \\
& \Leftrightarrow 1 \geq \theta_{n+1}+\sqrt{1-2 \theta_{n+1} \delta+\theta_{n+1}^{2} \sigma^{2}} .
\end{aligned}
$$

Consequently it follows from (8), (9) and (10) that for all $n \geq N_{0}$,

$$
\begin{aligned}
& \left\|T^{\left(\theta_{n+1}, \lambda_{n+1}\right)} u_{n}-T^{\left(\theta_{n+1}, \lambda_{n+1}\right)} u^{*}\right\| \\
& \leq \sqrt{1-2 \theta_{n+1} \delta+\theta_{n+1}^{2} \sigma^{2}}\left\|u_{n}-u^{*}\right\|+\left(\theta_{n+1}-\frac{1}{2} \lambda_{n+1} \mu \eta\right)\left\|u_{n}-u^{*}\right\| \\
& =\left(\theta_{n+1}+\sqrt{1-2 \theta_{n+1} \delta+\theta_{n+1}^{2} \sigma^{2}}-\frac{1}{2} \lambda_{n+1} \mu \eta\right)\left\|u_{n}-u^{*}\right\| \\
& \leq\left(1-\frac{1}{2} \lambda_{n+1} \mu \eta\right)\left\|u_{n}-u^{*}\right\| .
\end{aligned}
$$

Substituting (11) into (7), we obtain for all $n \geq N_{0}$,

$$
\left\|u_{n+1}-u^{*}\right\| \leq\left(1-\frac{1}{2} \lambda_{n+1} \mu \eta\right)\left\|u_{n}-u^{*}\right\|+\lambda_{n+1} \mu\left\|F\left(u^{*}\right)\right\| .
$$

By induction, it is easy to see that

$$
\left\|u_{n}-u^{*}\right\| \leq \max \left\{\max _{0 \leq i \leq N_{0}}\left\|u_{i}-u^{*}\right\|, \frac{2}{\eta}\left\|F\left(u^{*}\right)\right\|\right\} \quad n \geq 0
$$

Step 2. $\left\|u_{n+1}-T u_{n}\right\| \rightarrow 0 \quad n \rightarrow \infty$. Indeed by Step 1, $\left\{u_{n}\right\}$ is bounded and so are $\left\{T u_{n}-g\left(T u_{n}\right)\right\}$ and $\left\{F\left(T u_{n}\right)\right\}$. Hence

$$
\begin{aligned}
\left\|u_{n+1}-T u_{n}\right\| & =\left\|\theta_{n+1}\left(T u_{n}-g\left(T u_{n}\right)\right)-\lambda_{n+1} \mu F\left(T u_{n}\right)\right\| \\
& \leq \theta_{n+1}\left\|T u_{n}-g\left(T u_{n}\right)\right\|+\lambda_{n+1} \mu\left\|F\left(T u_{n}\right)\right\| \rightarrow 0 .
\end{aligned}
$$

Step 3. $\left\|u_{n+1}-u_{n}\right\| \rightarrow 0, n \rightarrow \infty$. Indeed by using the proof similar to that of (11), we can see that there exists an integer $N_{1} \geq 0$ such that for all $n \geq N_{1}$,

$$
\left\|T^{\left(\theta_{n+1}, \lambda_{n+1}\right)} u_{n}-T^{\left(\theta_{n+1}, \lambda_{n+1}\right)} u_{n-1}\right\| \leq\left(1-\frac{1}{2} \lambda_{n+1} \mu \eta\right)\left\|u_{n}-u_{n-1}\right\|
$$


which implies that for all $n \geq N_{1}$,

$$
\begin{aligned}
\left\|u_{n+1}-u_{n}\right\| & =\left\|T^{\left(\theta_{n+1}, \lambda_{n+1}\right)} u_{n}-T^{\left(\theta_{n}, \lambda_{n}\right)} u_{n-1}\right\| \\
& \leq\left\|T^{\left(\theta_{n+1}, \lambda_{n+1}\right)} u_{n}-T^{\left(\theta_{n+1}, \lambda_{n+1}\right)} u_{n-1}\right\|+\left\|T^{\left(\theta_{n+1}, \lambda_{n+1}\right)} u_{n-1}-T^{\left(\theta_{n}, \lambda_{n}\right)} u_{n-1}\right\| \\
& \leq\left(1-\frac{1}{2} \lambda_{n+1} \mu \eta\right)\left\|u_{n}-u_{n-1}\right\|+\left|\lambda_{n+1}-\lambda_{n}\right| \mu\left\|F\left(T u_{n-1}\right)\right\|+\gamma_{n+1}
\end{aligned}
$$

where $\gamma_{n+1}=\left|\theta_{n+1}-\theta_{n}\right|\left\|T u_{n-1}-g\left(T u_{n-1}\right)\right\|$. Putting

$$
M=\max \left\{\sup _{n \geq 0}\left\|F\left(T u_{n}\right)\right\|, \sup _{n \geq 0}\left\|T\left(u_{n}\right)-g\left(T u_{n}\right)\right\|\right\},
$$

we obtain

$$
\left\|u_{n+1}-u_{n}\right\| \leq\left(1-\frac{1}{2} \lambda_{n+1} \mu \eta\right)\left\|u_{n}-u_{n-1}\right\|+\left(\frac{1}{2} \lambda_{n+1} \mu \eta\right) \beta_{n+1}+\gamma_{n+1}
$$

where

$$
\beta_{n+1}=M \mu\left|\lambda_{n+1}-\lambda_{n}\right| /\left(\frac{1}{2} \lambda_{n+1} \mu \eta\right) \rightarrow 0 \quad\left(\operatorname{using}(\mathrm{L} 3)^{\prime}\right)
$$

and

$$
\sum_{n=N_{1}}^{\infty} \gamma_{n+1} \leq M \sum_{n=N_{1}}^{\infty}\left|\theta_{n+1}-\theta_{n}\right|<\infty \quad(\text { using (R3)) }
$$

By Lemma 2.1, we deduce that $\left\|u_{n+1}-u_{n}\right\| \rightarrow 0$.

Step 4. $\left\|u_{n}-T u_{n}\right\| \rightarrow 0$. This is an immediate consequence of Steps 2 and 3.

Step 5. $\lim \sup _{n \rightarrow \infty}\left\langle-F\left(u^{*}\right), u_{n}-u^{*}\right\rangle \leq 0$. To prove this, we pick a subsequence $\left\{u_{n_{i}}\right\}$ of $\left\{u_{n}\right\}$ so that

$$
\limsup _{n \rightarrow \infty}\left\langle-F\left(u^{*}\right), u_{n}-u^{*}\right\rangle=\lim _{i \rightarrow \infty}\left\langle-F\left(u^{*}\right), u_{n_{i}}-u^{*}\right\rangle
$$

Without loss of generality, we may further assume that $u_{n_{i}} \rightarrow \widetilde{u}$ weakly for some $\widetilde{u} \in H$. But by Lemma 2.2 and Step 4, we have $\widetilde{u} \in \operatorname{Fix}(T)=C$. Since $u^{*}$ is the unique solution of the 
$\mathrm{VI}(F, C)$, we obtain

$$
\limsup _{n \rightarrow \infty}\left\langle-F\left(u^{*}\right), u_{n}-u^{*}\right\rangle=\left\langle-F\left(u^{*}\right), \widetilde{u}-u^{*}\right\rangle \leq 0 .
$$

Step 6. $u_{n} \rightarrow u^{*}$ in norm. Indeed by Step 1 , we know that there exists an integer $N_{0} \geq 0$ such that for all $n \geq N_{0}$,

$$
\left\|T^{\left(\theta_{n+1}, \lambda_{n+1}\right)} u_{n}-T^{\left(\theta_{n+1}, \lambda_{n+1}\right)} u^{*}\right\| \leq\left(1-\frac{1}{2} \lambda_{n+1} \mu \eta\right)\left\|u_{n}-u^{*}\right\| .
$$

This together with Lemma 2.3 implies

$$
\begin{aligned}
\left\|u_{n+1}-u^{*}\right\|^{2} & =\left\|\left(T^{\left(\theta_{n+1}, \lambda_{n+1}\right)} u_{n}-T^{\left(\theta_{n+1}, \lambda_{n+1}\right)} u^{*}\right)+\left(T^{\left(\theta_{n+1}, \lambda_{n+1}\right)} u^{*}-u^{*}\right)\right\|^{2} \\
& \leq\left\|T^{\left(\theta_{n+1}, \lambda_{n+1}\right)} u_{n}-T^{\left(\theta_{n+1}, \lambda_{n+1}\right)} u^{*}\right\|^{2}+2\left\langle T^{\left(\theta_{n+1}, \lambda_{n+1}\right)} u^{*}-u^{*}, u_{n+1}-u^{*}\right\rangle \\
& \leq\left(1-\frac{1}{2} \lambda_{n+1} \mu \eta\right)\left\|u_{n}-u^{*}\right\|^{2}+2 \lambda_{n+1} \mu\left\langle-F\left(u^{*}\right), u_{n+1}-u^{*}\right\rangle \\
& \leq\left(1-\frac{1}{2} \lambda_{n+1} \mu \eta\right)\left\|u_{n}-u^{*}\right\|^{2}+\left(\frac{1}{2} \lambda_{n+1} \mu \eta\right) \cdot \frac{4}{\eta}\left\langle-F\left(u^{*}\right), u_{n+1}-u^{*}\right\rangle .
\end{aligned}
$$

An application of Lemma 2.1 combined with Step 5 yields that $\left\|u_{n}-u^{*}\right\| \rightarrow 0$.

Next we consider a more general case where

$$
C=\bigcap_{i=1}^{N} \operatorname{Fix}\left(T_{i}\right)
$$

with $N \geq 1$ an integer and $T_{i}: H \rightarrow H$ being nonexpansive for each $1 \leq i \leq N$.

We consider now another hybrid steepest-descent algorithm for solving the $\operatorname{GVI}(F, g, C)$ with $C$ defined above.

Algorithm (II). Let $\left\{\lambda_{n}\right\} \subset(0,1),\left\{\theta_{n}\right\} \subset(0,1]$ and $\mu \in\left(0,2 \eta / \kappa^{2}\right)$. Starting with an arbitrary initial guess $u_{0} \in H$, one can generate a sequence $\left\{u_{n}\right\}$ by the following iterative 
scheme

$$
\begin{aligned}
u_{n+1} & =T_{[n+1]}^{\left(\theta_{n+1}, \lambda_{n+1}\right)} u_{n} \\
& =\left(1+\theta_{n+1}\right) T_{[n+1]} u_{n}-\theta_{n+1} g\left(T_{[n+1]} u_{n}\right)-\lambda_{n+1} \mu F\left(T_{[n+1]} u_{n}\right), \quad n \geq 0 .
\end{aligned}
$$

Theorem 3.2. Let $\mu \in\left(0,2 \eta / \kappa^{2}\right)$, let conditions (L1), (L2) and (L4)' be satisfied and also let restrictions (R1), (R2) and (R4) be satisfied. Assume in addition that

$$
C=\bigcap_{i=1}^{N} \operatorname{Fix}\left(T_{i}\right)=\operatorname{Fix}\left(T_{1} T_{2} \ldots T_{N}\right)
$$

Suppose that $u^{*} \in \operatorname{Fix}(g)$. Then the sequence $\left\{u_{n}\right\}$ generated by algorithm (12) converges in norm to $u^{*}$ which is a solution of the $\operatorname{GVI}(F, g, C)$.

Proof. We shall again divide the proof into several steps.

Step 1. $\left\{u_{n}\right\}$ is bounded. Indeed, as in Step 1 of the proof of Theorem 3.1, we know that there exists an integer $N_{0} \geq 0$ such that for all $n \geq N_{0}$,

$$
\left\|T_{[n+1]}^{\left(\theta_{n+1}, \lambda_{n+1}\right)} u_{n}-T_{[n+1]}^{\left(\theta_{n+1}, \lambda_{n+1}\right)} u^{*}\right\| \leq\left(1-\frac{1}{2} \lambda_{n+1} \mu \eta\right)\left\|u_{n}-u^{*}\right\|
$$

from which we obtain (note that $T_{[n]}^{\left(\theta_{n}, \lambda_{n}\right)} u^{*}=u^{*}-\lambda_{n} \mu F\left(u^{*}\right)$ for all $n \geq 1$ )

$$
\begin{aligned}
\left\|u_{n+1}-u^{*}\right\| & =\left\|T_{[n+1]}^{\left(\theta_{n+1}, \lambda_{n+1}\right)} u_{n}-u^{*}\right\| \\
& \leq\left\|T_{[n+1]}^{\left(\theta_{n+1}, \lambda_{n+1}\right)} u_{n}-T_{[n+1]}^{\left(\theta_{n+1}, \lambda_{n+1}\right)} u^{*}\right\|+\left\|T_{[n+1]}^{\left(\theta_{n+1}, \lambda_{n+1}\right)} u^{*}-u^{*}\right\| \\
& \leq\left(1-\frac{1}{2} \lambda_{n+1} \mu \eta\right)\left\|u_{n}-u^{*}\right\|+\lambda_{n+1} \mu\left\|F\left(u^{*}\right)\right\| .
\end{aligned}
$$

By induction, it is easy to see that

$$
\left\|u_{n}-u^{*}\right\| \leq \max \left\{\max _{0 \leq i \leq N_{0}}\left\|u_{i}-u^{*}\right\|, \frac{2}{\eta}\left\|F\left(u^{*}\right)\right\|\right\} \quad n \geq 0 .
$$


Step 2. $\left\|u_{n+1}-T u_{n}\right\| \rightarrow 0, n \rightarrow \infty$. Indeed, by Step 1, $\left\{u_{n}\right\}$ is bounded and so are $\left\{T_{[n+1]} u_{n}-g\left(T_{[n+1]} u_{n}\right)\right\}$ and $\left\{F\left(T_{[n+1]} u_{n}\right)\right\}$. Hence

$$
\begin{aligned}
\left\|u_{n+1}-T u_{n}\right\| & =\left\|\theta_{n+1}\left(T_{[n+1]} u_{n}-g\left(T_{[n+1]} u_{n}\right)\right)-\lambda_{n+1} \mu F\left(T_{[n+1]} u_{n}\right)\right\| \\
& \leq \theta_{n+1}\left\|T_{[n+1]} u_{n}-g\left(T_{[n+1]} u_{n}\right)\right\|+\lambda_{n+1} \mu\left\|F\left(T_{[n+1]} u_{n}\right)\right\| \rightarrow 0 .
\end{aligned}
$$

Step 3. $\left\|u_{n+N}-u_{n}\right\| \rightarrow 0$. As a matter of fact, by using the proof similar to that of (11), we can see that there exists an integer $N_{1} \geq 0$ such that for all $n \geq N_{1}$,

$$
\left\|T_{[n+N]}^{\left(\theta_{n+N}, \lambda_{n+N}\right)} u_{n+N-1}-T_{[n+N]}^{\left(\theta_{n+N}, \lambda_{n+N}\right)} u_{n-1}\right\| \leq\left(1-\frac{1}{2} \lambda_{n+N} \mu \eta\right)\left\|u_{n+N-1}-u_{n-1}\right\| .
$$

Noticing $T_{[n+N]}=T_{[n]}$ and utilizing (14), we deduce that for all $n \geq N_{1}$,

$$
\begin{aligned}
& \left\|u_{n+N}-u_{n}\right\| \\
& =\left\|T_{[n+N]}^{\left(\theta_{n+N}, \lambda_{n+N}\right)} u_{n+N-1}-T_{[n]}^{\left(\theta_{n}, \lambda_{n}\right)} u_{n-1}\right\| \\
& \leq\left\|T_{[n+N]}^{\left(\theta_{n+N}, \lambda_{n+N}\right)} u_{n+N-1}-T_{[n+N]}^{\left(\theta_{n+N}, l a m b d a_{n+N}\right)} u_{n-1}\right\|+\left\|T_{[n+N]}^{\left(\theta_{n+N}, \lambda_{n+N}\right)} u_{n-1}-T_{[n]}^{\left(\theta_{n}, \lambda_{n}\right)} u_{n-1}\right\| \\
& \leq\left(1-\frac{1}{2} \lambda_{n+N} \mu \eta\right)\left\|u_{n+N-1}-u_{n-1}\right\|+\left|\lambda_{n+N}-\lambda_{n}\right| \mu\left\|F\left(T_{[n]} u_{n-1}\right)\right\|+\gamma_{n}
\end{aligned}
$$

where $\gamma_{n}=\left|\theta_{n+N}-\theta_{n}\right||| T_{[n]} u_{n-1}-g\left(T_{[n]} u_{n-1}\right) \|$. Putting

$$
M=\max \left\{\sup _{n \geq 1}\left\|F\left(T_{[n]} u_{n-1}\right)\right\|, \sup _{n \geq 1}\left\|T_{[n]} u_{n-1}-g\left(T_{[n]} u_{n-1}\right)\right\|\right\}
$$

we obtain

$$
\left\|u_{n+N}-u_{n}\right\| \leq\left(1-\frac{1}{2} \lambda_{n+N} \mu \eta\right) \| u_{n+N-1}-u_{n-1} \mid+\left(\frac{1}{2} \lambda_{n+N} \mu \eta\right) \beta_{n}+\gamma_{n}
$$

where

$$
\beta_{n}=M \mu\left|\lambda_{n+N}-\lambda_{n}\right| /\left(\frac{1}{2} \lambda_{n+N} \mu \eta\right) \rightarrow 0 \quad\left(\operatorname{using}(\mathrm{L} 4)^{\prime}\right)
$$

and

$$
\sum_{n=N_{1}}^{\infty} \gamma_{n} \leq M \sum_{n=N_{1}}^{\infty}\left|\theta_{n+N}-\theta_{n}\right|<\infty . \quad(\text { using }(\mathrm{R} 4))
$$


Now we apply Lemma 2.1 to see that $\left\|u_{n+N}-u_{n}\right\| \rightarrow 0$.

Step 4. $u_{n}-T_{[n+N]} \ldots T_{[n+1]} u_{n} \rightarrow 0$ in norm. Indeed noting that each $T_{i}$ is nonexpansive and using Step 2, we get the finite table

$$
\begin{aligned}
& u_{n+N}-T_{[n+N]} u_{n+N-1} \rightarrow 0, \\
& T_{[n+N]} u_{n+N-1}-T_{[n+N]} T_{[n+N-1]} u_{n+N-2} \rightarrow 0, \\
& \vdots \\
& T_{[n+N]} \ldots T_{[n+2]} u_{n+1}-T_{[n+N]} \ldots T_{[n+1]} u_{n} \rightarrow 0 .
\end{aligned}
$$

Adding up this table yields that $u_{n}-T_{[n+N]} \ldots T_{[n+1]} u_{n} \rightarrow 0$ in norm.

Step 5. $\lim \sup _{n \rightarrow \infty}\left\langle-F\left(u^{*}\right), u_{n}-u^{*}\right\rangle \leq 0$. To see this we pick a subsequence $\left\{u_{n_{i}}\right\}$ of $\left\{u_{n}\right\}$ such that

$$
\limsup _{n \rightarrow \infty}\left\langle-F\left(u^{*}\right), u_{n}-u^{*}\right\rangle=\lim _{i \rightarrow \infty}\left\langle-F\left(u^{*}\right), u_{n_{i}}-u^{*}\right\rangle
$$

Since $\left\{u_{n}\right\}$ is bounded, we may also assume that $u_{n_{i}} \rightarrow \widetilde{u}$ weakly for some $\widetilde{u} \in H$. Since the pool of mappings $\left\{T_{i}: 1 \leq i \leq N\right\}$ is finite, we may further assume (passing to a further subsequence if necessary) that for some integer $k \in\{1,2, \ldots, N\}, T_{\left[n_{i}\right]} \equiv T_{k} \forall i \geq 1$. Then it follows from Step 4 that $u_{n_{i}}-T_{[i+N]} \ldots T_{[i+1]} u_{n_{i}} \rightarrow 0$. Hence by Lemma 2.2, we deduce that

$$
\widetilde{u} \in \operatorname{Fix}\left(T_{[i+N]} \ldots T_{[i+1]}\right) .
$$

which together with assumption (13) implies that $\widetilde{u} \in C$. Now since $u^{*}$ solves the $\operatorname{VI}(F, C)$, 
we obtain

$$
\limsup _{n \rightarrow \infty}\left\langle-F\left(u^{*}\right), u_{n}-u^{*}\right\rangle=\left\langle-F\left(u^{*}\right), \widetilde{u}-u^{*}\right\rangle \leq 0 .
$$

Step 6. $u_{n} \rightarrow u^{*}$ in norm. Indeed by Step 1, we know that there exists an integer $N_{0} \geq 0$ such that for all $n \geq N_{0}$,

$$
\left\|T_{[n+1]}^{\left(\theta_{n+1}, \lambda_{n+1}\right)} u_{n}-T_{[n+1]}^{\left(\theta_{n+1}, \lambda_{n+1}\right)} u^{*}\right\| \leq\left(1-\frac{1}{2} \lambda_{n+1} \mu \eta\right)\left\|u_{n}-u^{*}\right\| .
$$

This together with Lemma 2.3 implies

$$
\begin{aligned}
\left\|u_{n+1}-u^{*}\right\|^{2} & =\left\|\left(T_{[n+1]}^{\left(\theta_{n+1}, \lambda_{n+1}\right)} u_{n}-T_{[n+1]}^{\left(\theta_{n+1}, \lambda_{n+1}\right)} u^{*}\right)+\left(T_{[n+1]}^{\left(\theta_{n+1}, \lambda_{n+1}\right)} u^{*}-u^{*}\right)\right\|^{2} \\
& \leq\left\|T_{[n+1]}^{\left(\theta_{n+1}, \lambda_{n+1}\right)} u_{n}-T_{[n+1]}^{\left(\theta_{n+1}, \lambda_{n+1}\right)} u^{*}\right\|^{2}+2\left\langle T_{[n+1]}^{\left(\theta_{n+1}, \lambda_{n+1}\right)} u^{*}-u^{*}, u_{n+1}-u^{*}\right\rangle \\
& \leq\left(1-\frac{1}{2} \lambda_{n+1} \mu \eta\right)\left\|u_{n}-u^{*}\right\|^{2}+2 \lambda_{n+1} \mu\left\langle-F\left(u^{*}\right), u_{n+1}-u^{*}\right\rangle \\
& \leq\left(1-\frac{1}{2} \lambda_{n+1} \mu \eta\right)\left\|u_{n}-u^{*}\right\|^{2}+\left(\frac{1}{2} \lambda_{n+1} \mu \eta\right) \cdot \frac{4}{\eta}\left\langle-F\left(u^{*}\right), u_{n+1}-u^{*}\right\rangle .
\end{aligned}
$$

By Lemma 2.1 and Step 5, we obtain that $\left\|u_{n}-u^{*}\right\| \rightarrow 0$.

Remark 3.1. Recall that a self-mapping $T$ of a nonempty closed convex subset $K$ of a Hilbert space $H$ is said to be attracting nonexpansive (Refs. 1-2) if $T$ is nonexpansive and if $\|T x-p\|<\|x-p\|$ for $x, p \in K$ with $x \notin \operatorname{Fix}(T)$ and $p \in \operatorname{Fix}(T)$. Recall also that $T$ is firmly nonexpansive (Refs. 1-2) if $\langle x-y, T x-T y\rangle \geq\|T x-T y\|^{2}$ for all $x, y \in K$. We observe that any firmly nonexpansive mappings is attracting nonexpansive. It is known that assumption (13) in Theorem 3.2 is automatically satisfied if each $T_{i}$ is attracting nonexpansive. Since a projection is firmly nonexpansive, we have the following consequence of Theorem 3.2.

Corollary 3.1. Let $\mu \in\left(0,2 \eta / \kappa^{2}\right)$ and conditions (L1), (L2), (L4)' be satisfied. Also let restrictions (R1), (R2) and (R4) be satisfied. Let $u_{0} \in H$ and let the sequence $\left\{u_{n}\right\}$ be 
generated by the iterative algorithm

$$
u_{n+1}:=\left(1+\theta_{n+1}\right) P_{[n+1]} u_{n}-\theta_{n+1} g\left(P_{[n+1]} u_{n}\right)-\lambda_{n+1} \mu F\left(P_{[n+1]} u_{n}\right), \quad n \geq 0,
$$

where $P_{k}=P_{C_{k}}, 1 \leq k \leq N$. Suppose that the unique solution $u^{*}$ of $\operatorname{VI}(F, C)$ is also a fixed point of $g$ where $C=\bigcap_{k=1}^{N} C_{k}$. Then $\left\{u_{n}\right\}$ converges strongly to $u^{*}$ which is a solution of $G V I(F, g, C)$.

In particular, let $m_{0}$ be an integer satisfying $m_{0} \geq\left(\sigma^{2}-1\right)^{2} / 4(\delta-1)^{2}$. Then the sequence $\left\{u_{n}\right\}$ determined by the algorithm

$$
u_{n+1}:=\left(1+\frac{1}{\sqrt{n+m_{0}+1}}\right) P_{[n+1]} u_{n}-\frac{1}{\sqrt{n+m_{0}+1}} g\left(P_{[n+1]} u_{n}\right)-\frac{\mu}{n+1} F\left(P_{[n+1]} u_{n}\right), \quad n \geq 0
$$

converges in norm to a solution $u^{*}$ of the $\operatorname{GVI}(F, g, C)$.

\section{Applications to Constrained Generalized Pseudoinverse}

Let $K$ be a nonempty closed convex subset of a real Hilbert space $H$. Let $A$ be a bounded linear operator on $H$. Given an element $b \in H$, consider the minimization problem

$$
\min _{x \in K}\|A x-b\|^{2}
$$

Let $S_{b}$ denote the solution set. Then, $S_{b}$ is closed convex. It is known that $S_{b}$ is nonempty if and only if $P_{\overline{A(K)}}(b) \in A(K)$. In this case $S_{b}$ has a unique element with minimum norm; that is, there exists a unique point $\hat{x} \in S_{b}$ satisfying

$$
\|\hat{x}\|^{2}=\min \left\{\|x\|^{2}: x \in S_{b}\right\}
$$


Definition 4.1. (See Ref. 4.) The $K$-constrained pseudoinverse of $A$ (symbol $\hat{A}_{K}$ ) is defined as

$$
D\left(\hat{A}_{K}\right)=\left\{b \in H: P_{\overline{A(K)}}(b) \in A(K)\right\}, \quad \hat{A}_{K}(b)=\hat{x} \quad \text { and } \quad b \in D\left(\hat{A}_{K}\right)
$$

where $\hat{x} \in S_{b}$ is the unique solution to (16).

Now we recall the $K$-constrained generalized pseudoinverse of $A$.

Let $\theta: H \rightarrow R$ be a differentiable convex function such that $\theta^{\prime}$ is a $\kappa$-Lipschitzian and $\eta$-strongly monotone operator for some $\kappa>0$ and $\eta>0$. Under these assumptions, there exists a unique point $\hat{x}_{0} \in S_{b}$ for $b \in D\left(\hat{A}_{K}\right)$ such that

$$
\theta\left(\hat{x}_{0}\right)=\min \left\{\theta(x): x \in S_{b}\right\}
$$

Definition 4.2. (See Ref. 16.) The $K$-constrained generalized pseudoinverse of $A$ associated with $\theta\left(\right.$ symbol $\left.\hat{A}_{K, \theta}\right)$ is defined as

$$
D\left(\hat{A}_{K, \theta}\right)=D\left(\hat{A}_{K}\right), \quad \hat{A}_{K, \theta}(b)=\hat{x}_{0}, \quad \text { and } \quad b \in D\left(\hat{A}_{K, \theta}\right)
$$

where $\hat{x}_{0} \in S_{b}$ is the unique solution to (17). Note that if $\theta(x)=\|x\|^{2} / 2$, then the $K$ constrained generalized pseudoinverse $\hat{A}_{K, \theta}$ of $A$ associated with $\theta$ reduces to the $K$-constrained pseudoinverse $\hat{A}_{K}$ of $A$ in Definition 4.1 .

We now apply the results in Section 3 to construct the $K$-constrained generalized pseudoinverse $\hat{A}_{K, \theta}$ of $A$. But first observe that $\widetilde{x} \in K$ solves the minimization problem (15) if and 
only if there holds the following optimality condition:

$$
\left\langle A^{*}(A \widetilde{x}-b), x-\widetilde{x}\right\rangle \geq 0, \quad x \in K,
$$

where $A^{*}$ is the adjoint of $A$. This is equivalent to for each $\lambda>0$,

$$
\left\langle\left[\lambda A^{*} b+\left(I-\lambda A^{*} A\right) \widetilde{x}\right]-\widetilde{x}, \widetilde{x}-x\right\rangle \geq 0, \quad x \in K,
$$

or

$$
P_{K}\left(\lambda A^{*} b+\left(I-\lambda A^{*} A\right) \widetilde{x}\right)=\widetilde{x} .
$$

Define a mapping $T: H \rightarrow H$ by

$$
T x=P_{K}\left(A^{*} b+\left(I-\lambda A^{*} A\right) x\right), \quad x \in H .
$$

Lemma 4.1. (See Ref. 16.) If $\lambda \in\left(0,2\|A\|^{-2}\right)$ and if $b \in D\left(\hat{A}_{K}\right)$, then $T$ is attracting nonexpansive and $\operatorname{Fix}(T)=S_{b}$.

Theorem 4.1. Assume that $0<\mu<2 \eta / \kappa^{2}$. Assume that the restrictions (R1), (R2) and (R3) hold for $\left\{\theta_{n}\right\}$ and also that the control conditions (L1), (L2), and (L3)' hold for $\left\{\lambda_{n}\right\}$. Given an initial guess $u_{0} \in H$, let $\left\{u_{n}\right\}$ be the sequence generated by the algorithm

$$
u_{n+1}=\left(1+\theta_{n+1}\right) T u_{n}-\theta_{n+1} g\left(T u_{n}\right)-\lambda_{n+1} \mu \theta^{\prime}\left(T u_{n}\right), \quad n \geq 0
$$

where $T$ is given in (19). Suppose that the unique solution $\hat{x}_{0}$ of (17) is also a fixed point of g. Then $\left\{u_{n}\right\}$ strongly converges to $\hat{A}_{K, \theta}(b)$. 
Proof. The minimization problem (17) is equivalent to the following variational inequality problem:

$$
\left\langle\theta^{\prime}\left(\hat{x}_{0}\right), x-\hat{x}_{0}\right\rangle \geq 0, \quad x \in S_{b}
$$

Since $\operatorname{Fix}(T)=S_{b}$ and $\theta^{\prime}$ is $\kappa$-Lipschitzian and $\eta$-strongly monotone, using Theorem 3.1 with $F:=\theta^{\prime}$, we infer that $\left\{u_{n}\right\}$ converges in norm to $\hat{x}_{0}=\hat{A}_{K, \theta}(b)$.

Lemma 4.2. (See Refs. 1-2.) Assume that $N$ is a positive integer and assume that $\left\{T_{i}\right\}_{i=1}^{N}$ are $N$ attracting nonexpansive mappings on $H$ having a common fixed point. Then

$$
\bigcap_{i=1}^{N} \operatorname{Fix}\left(T_{i}\right)=\operatorname{Fix}\left(T_{1} T_{2} \ldots T_{N}\right)
$$

Now assume that $\left\{S_{b}^{1}, \ldots, S_{b}^{N}\right\}$ is a family of $N$ closed convex subsets of $K$ such that $S_{b}=\bigcap_{i=1}^{N} S_{b}^{i}$. For each $1 \leq i \leq N$, we define $T_{i}: H \rightarrow H$ by

$$
T_{i} x=P_{S_{b}^{i}}\left(A^{*} b+\left(I-\lambda A^{*} A\right) x\right) \quad x \in H
$$

where $P_{S_{b}^{i}}$ is the projection from $H$ onto $S_{b}^{i}$.

Theorem 4.2. Let $\mu \in\left(0,2 \eta / \kappa^{2}\right)$, let conditions (L1), (L2) and (L4)' be satisfied and also let restrictions (R1), (R2) and (R4) be satisfied. Let $u_{0} \in H$. Suppose that the unique solution $\hat{x}_{0}$ of $(17)$ is also a fixed point of $g$. Then the sequence $\left\{u_{n}\right\}$ generated by the algorithm

$$
u_{n+1}=T_{[n+1]}^{\left(\theta_{n+1}, \lambda_{n+1}\right)} u_{n}=\left(1+\theta_{n+1}\right) T_{[n+1]} u_{n}-\theta_{n+1} g\left(T_{[n+1]} u_{n}\right)-\lambda_{n+1} \mu \theta^{\prime}\left(T_{[n+1]} u_{n}\right), \quad n \geq 0
$$

converges in norm to $\hat{A}_{K, \theta}(b)$. 
Proof. In the proof of (Ref. 16, Theorem 4.2), Xu and Kim have proved that

$$
S_{b}=\operatorname{Fix}(T)=\bigcap_{i=1}^{N} \operatorname{Fix}\left(T_{i}\right) .
$$

By Lemmas 4.1 and 4.2, we see that assumption (13) in Theorem 3.2 holds. On account of (23), Theorem 3.2 ensures that the sequence $\left\{u_{n}\right\}$ generated by (22) converges strongly to the unique solution $\hat{x}_{0}=\hat{A}_{K, \theta}(b)$ of $(21)$.

\section{References}

1. BAUSCHKE, H. H., The Approximation of Fixed Points of Compositions of Nonexpansive Mappings in Hilbert Spaces, Journal of Mathematical Analysis and Applications, Vol. 202, pp. 150-159, 1996.

2. BAUSCHKE, H. H., and BORWEIN, J. M., On Projection Algorithms for Solving Convex Feasibility Problems, SIAM Review, Vol. 38, pp. 367-426, 1996.

3. DEUTSCH, F., and YAMADA, I., Minimizing Certain Convex Functions over the Intersection of the Fixed-Point Sets of Nonexpansive Mappings, Numerical Functional Analysis and Optimization, Vol. 19, pp. 33-56, 1998.

4. ENGL, H. W., HANKE, M., and NEUBAUER, A., Regularization of Inverse Problems, Kluwer, Dordrecht, Holland, 2000. 
5. GEOBEL, K., and KIRK, W. A., Topics on Metric Fixed-Point Theory, Cambridge University Press, Cambridge, England, 1990.

6. GLOWINSKI, R., Numerical Methods for Nonlinear Variational Problems, Springer, New York, NY, 1984.

7. JAILLET, P., LAMBERTON, D., and LAPEYRE, B., Variational Inequalities and the Pricing of American Options, Acta Applicandae Mathematicae, Vol. 21, pp. 263-289, 1990.

8. KINDERLEHRER, D., and STAMPACCHIA, G., An Introduction to Variational Inequalities and Their Applications, Academic Press, New York, NY, 1980.

9. KONNOV, I., Combined Relaxation Methods for Variational Inequalities, Springer, Berlin, Germany, 2001.

10. LIONS, P. L., Approximation de Points Fixes de Contractions, Comptes Rendus de L'Academie des Sciences de Paris, Vol. 284, pp. 1357-1359, 1977.

11. ODEN, J. T., Qualitative Methods on Nonlinear Mechanics, Prentice-Hall, Englewood Cliffs, New Jersey, 1986.

12. PANG, J. S., and YAO, J. C., On a Generalization of a Normal Map and Equations, SIAM Journal on Control and Optimization, Vol. 33, pp. 168-184, 1995. 
13. WitTmann, R., Approximation of Fixed Points of Nonexpansive Mappings, Archiv der Mathematik, Vol. 58, pp. 486-491,1992.

14. XU, H. K., Iterative algorithms for nonlinear operators, Journal of London Mathematical Society, Vol. 66, No. 2, pp. 240-256, 2002.

15. Xu, H. K., An Iterative Approach to Quadratic Optimization, Journal of Optimization Theory and Applications, Vol. 116, pp. 659-678, 2003.

16. XU, H. K., and KIM, T. H., Convergence of Hybrid Steepest-Descent Methods for Variational Inequalities, Journal of Optimization Theory and Applications, Vol. 119, pp. 185-201, 2003.

17. YAMADA, I., The Hybrid Steepest-Descent Mathod for Variational Inequality Problems over the Intersection of the Fixed-Point Sets of Nonexpansive Mappings, Inherently Parallel Algorithms in Feasibility and Optimization and Their Applications, Edited by D. Butnariu, Y. Censor, and S. Reich, North-Holland, Amsterdam, Holland, pp. 473$504,2001$.

18. YAO, J. C., Variational Inequalities With generalized Monotone Operators, Mathematics of Operational Research, Vol. 49, pp. 691-705, 1994.

19. ZENG, L. C., Completely Generalized Strongly Nonlinear Quasi-Complementarity Problems in Hilbert Spaces, Journal of Mathematical Analysis and Applications, Vol. 193, 
pp. 706-714, 1995.

20. ZENG, L. C., Iterative Algorithm for Finding Approximate Solutions to Completely Generalized Strongly Nonlinear Quasivariational Inequalities, Journal of Mathematical Analysis and Applications, Vol. 201, pp. 180-194, 1996.

21. ZENG, L. C., On a General Projection Algorithm for Variational Inequalities, Journal of Optimization Theory and Applications, Vol. 97, pp. 229-235, 1998. 